\title{
Osteogenesis induced by frizzled-related protein (FRZB) is linked to the netrin-like domain
}

\author{
Sarah Thysen ${ }^{1}$, Frederic Cailotto ${ }^{1}$ and Rik Lories ${ }^{1,2}$
}

\begin{abstract}
Abnormal Wnt signaling is associated with bone mass disorders. Frizzled-related protein (FRZB, also known as secreted frizzled-related protein-3 (SFRP3)) is a Wnt modulator that contains an amino-terminal cysteine-rich domain (CRD) and a carboxy-terminal Netrin-like (NTN) motif. Frzb ${ }^{-1-}$ mice show increased cortical thickness. However, the direct effect of FRZB on osteogenic differentiation and the involvement of the structural domains herein are not fully understood. In this study, we observed that stable overexpression of Frzb in MC3T3-E1 cells increased calcium deposition and osteoblast markers compared with control. Western blot analysis showed that the increased osteogenesis was associated with reduced canonical, but increased non-canonical Wnt signaling. On the contrary, loss of Frzb induced the opposite effects on osteogenesis and Wnt signaling. To translationally validate the positive effects of FRZB on primary human cells, we treated human periosteal and human bone marrow stromal cells with conditioned medium from MC3T3-E1 cells overexpressing Frzb and observed an increase in Alizarin red staining. We further studied the effect of the domains. $F r z b_{\text {NTN }}$ overexpression induced similar effects on osteogenesis as full-length Frzb, whereas Frzb $b_{C R D}$ overexpressing cells mimicked loss of Frzb experiments. The CRD is considered as the Wnt binding domain, but the NTN domain also has important effects on bone biology. FRZB and other SFRPs or their specific domains may hold surprising potential as therapeutics for bone and joint disorders considering that excess of SFRPs has effects that are not expected under physiological, endogenous expression conditions.

Laboratory Investigation (2016) 96, 570-580; doi:10.1038/labinvest.2016.38; published online 29 February 2016
\end{abstract}

The Wnt signaling pathway is a key regulator of development, tissue growth, homeostasis, and disease. Animal models and human genetic syndromes link abnormal Wnt signaling with high and low bone mass. ${ }^{1,2}$ The Wnt family is a large group of lipid-modified secreted glycoproteins. Mammals have $19 \mathrm{Wnt}$ homologs composed of 350-400 amino acids that share an $\mathrm{N}$-terminal signal sequence for secretion followed by a nearly invariant pattern of 22-24 cysteines and several $\mathrm{N}$-glycosylation sites. ${ }^{3,4}$ Wnts are palmytoylated intracellularly by the enzyme porcupine. The presence of the lipid side chain affects solubility and signaling range.

Active Wnt signaling typically involves the canonical or $\beta$-catenin-dependent cascade. In the absence of Wnt ligand, Frizzled (Fz) receptor, and low-density lipoprotein receptorrelated protein- $5 / 6$ coreceptor interaction, $\beta$-catenin is caught in a molecular destruction complex, phosphorylated and degraded by the proteasome. Upon Wnt-receptor interaction, the destruction complex is tethered away toward the receptor complex or disassembles, $\beta$-catenin accumulates in the cell, translocates to the nucleus, and associates with transcription factors of the T-cell factor/lymphoid enhancer factor family. Wnts also signal through $\beta$-catenin-independent pathways. The planar cell polarity pathway activates GTPases of the RHO family and c-Jun $\mathrm{N}$-terminal kinase to regulate the cytoskeleton and cell polarity, while the $\mathrm{Wnt} / \mathrm{Ca}^{2+}$ pathway stimulates heterotrimeric $G$ proteins, intracellular calcium release and activation of various calcium-sensitive enzymes such as protein kinase $\mathrm{C}$, calcium-calmodulin-dependent kinase II (CamKII), and phosphatase calcineurin. ${ }^{3,5}$

The secreted frizzled-related protein (SFRP)-family comprises five secreted molecules (SFRP1-5) and can modulate both canonical and non-canonical Wnt cascades. Every SFRP contains an amino-terminal cysteine-rich domain (CRD) and a carboxy-terminal Netrin-like (NTN) motif. The CRD shares structural homology with the $\mathrm{Fz}$ receptor, and is therefore considered as the Wnt binding domain. The NTN domain shows homology to the axonal guidance protein Netrin, and is also found in tissue inhibitors of metalloproteinases (TIMPs),

\footnotetext{
${ }^{1}$ Laboratory of Tissue Homeostasis and Disease, Department of Development and Regeneration, Skeletal Biology and Engineering Research Center, KU Leuven, Belgium and ${ }^{2}$ Division of Rheumatology, University Hospitals Leuven, Leuven, Belgium

Correspondence: Professor R Lories MD, PhD, Laboratory of Tissue Homeostasis and Disease, SBE Research Centre, O\&N1_Box 813, Herestraat 49, Leuven B3000, Belgium. E-mail: Rik.Lories@uz.kuleuven.be
}

Received 27 October 2014; revised 5 January 2016; accepted 25 January 2016 
complement proteins, and type I procollagen C-proteinase enhancer proteins (PCOLCEs). Until now, the exact role of the NTN domain in SFRP function remains unclear. ${ }^{6,7}$

SFPRs were originally identified as extracellular ligandbinding inhibitors of the Wnt signaling cascade. Recent data suggest that SFRPs have a broad range of biological activities, including negative and positive modulation of Wnt signaling 8,9 and interactions with molecules unrelated to Wnt signaling. ${ }^{7}$ Deletions of Frizzled-related protein (Frzb (Sfrp3)) in mice lead to increased cortical thickness without changes in the trabecular bone. ${ }^{10}$ Here, we aimed to directly assess the effect of FRZB on osteogenic differentiation and to elucidate the involvement of the CRD and NTN domain.

\section{MATERIALS AND METHODS \\ Molecular Constructs}

The pcDNA3.1-full-length Frzb, pcDNA3.1-Frzb $\triangle C R D_{\mathrm{AA} 39-145 \text { (7C) }}$ (referred to as Frzb $b_{\mathrm{NTN}}$ ), and pcDNA3.1-Frzb $\Delta$ NTN $_{\mathrm{AA} 160-316}$ (referred to as Frzb $_{\mathrm{CRD}}$ ) constructs were earlier described. ${ }^{11}$

\section{Cell Culture}

Human periosteum-derived cells (hPDCs) and human bone marrow stromal cells (hBMSCs) (a kind gift from C Verfaillie, $\mathrm{KU}$ Leuven) were isolated as described earlier. ${ }^{12,13} \mathrm{hPDCs}$, hBMSCs, and MC3T3-E1 cells were cultured in maintenance medium DMEM (Gibco, Merelbeke, Belgium) containing $10 \%$ fetal bovine serum (FBS, Gibco), $1 \%$ antibioticantimycotic ( $\mathrm{AB}$, Invitrogen, Merelbeke, Belgium), and $1 \%$ sodium pyruvate (SP, Invitrogen).

\section{Generation of Stably Transfected MC3T3-E1 Cell Lines}

MC3T3-E1 cells were transfected with control pcDNA3.1+, pcDNA3.1-Frzb, pcDNA3.1-Frz $b_{C R D}$, or pcDNA3.1-Frzb $b_{N T N}$ constructs using lipid-based agent Fugene HD (Roche Diagnostic, Vilvoorde, Belgium). Selection was initiated $24 \mathrm{~h}$ after transfection by supplementing the maintenance medium with $0.1 \mathrm{mg} / \mathrm{ml}$ geneticin (Invitrogen) for pcDNA3.1-Frz $b_{C R D}$ and $1 \mathrm{mg} / \mathrm{ml}$ for all other constructs. Clones were picked after 14 days of selection. Three different antibiotic-resistant clonal colonies of each condition were isolated and grown independently. Overexpression levels were assessed by quantitative RT-PCR (Q-PCR). Silencing of Frzb was performed using a pGIPZ-shmiRNA construct directed against mouse Frzb (Thermo Scientific, Aalst, Belgium); and a non-interfering pGIPZ vector (Thermo Scientific) was used as a control. MC3T3-E1 cells were transfected using Arrest-In transfection reagent (Thermo Scientific) and after $24 \mathrm{~h}$, selection with $1 \mu \mathrm{g} / \mathrm{ml}$ puromycin (Invitrogen) was initiated and continued for 7 days. Three different antibiotic-resistant clonal colonies of each condition were isolated and grown independently. Knockdown efficiency was assessed by Q-PCR. Antibiotic pressure was maintained for the whole duration of the experiments.

\section{Osteogenic Differentiation}

Stably transfected MC3T3-E1 cells were seeded at $2.5 \times 10^{4}$ cells/well in 6-well culture plates (Thermo Scientific) in maintenance medium. At day 1 , medium was changed to differentiation medium ( $\alpha$-Minimum Essential Medium Eagle $(\alpha$-MEM) (Gibco) containing 10\% FBS, $1 \%$ AB, $10 \mathrm{mM}$ $\beta$-glycerophosphate and $50 \mu \mathrm{g} / \mathrm{ml}$ L-ascorbic acid-2-phosphate). The medium was refreshed every other day for 21 days. hPDCs were seeded at $1.5 \times 10^{4}$ cells/well in 12 -well culture plates (Thermo Scientific, Nunc) in maintenance medium. At day 1 , medium was changed to differentiation medium consisting of $\alpha$-MEM supplemented with $10 \%$ FBS, $1 \% \mathrm{AB}, 10 \mathrm{mM} \beta$-glycerophosphate, $50 \mu \mathrm{g} / \mathrm{ml}$ L-ascorbic acid-2-phosphate and $0.1 \mathrm{nM}$ dexamethasone (Sigma-Aldrich, Bornem, Belgium). Medium was changed every other day for 28 days of differentiation. Cells were stored at different time points for Alizarin red staining, RNA, protein extraction, and alkaline phosphatase (ALP) activity assay. All conditions were performed in triplicate. For competition experiments with recombinant $\mathrm{mFrzb}$, the differentiation medium was supplemented with $100 \mathrm{ng} / \mathrm{ml}$ recombinant mFrzb (R\&D Systems) for 14 days. For experiments under the stimulation of canonical or non-canonical Wnts, we treated the MC3T3-E1 cells with $100 \mathrm{ng} / \mathrm{ml}$ recombinant $\mathrm{mWnt} 3 \mathrm{a}$ (R\&D systems) or mWnt5a (R\&D systems) during 14 days of differentiation.

\section{Conditioned Medium Experiment}

Supernatant (referred to as conditioned medium (CM)) was harvested every other day from confluent T75 culture flasks of MC3T3-E1 cells stably transfected with pcDNA3.1+ control vector or pcDNA3.1-Frzb. The supernatant was cleared by centrifugation at 3500 r.p.m. for $10 \mathrm{~min}$, and supplemented with $10 \mathrm{mM} \beta$-glycerophosphate and $50 \mu \mathrm{g} / \mathrm{ml} \mathrm{L}$-ascorbic acid-2-phosphate. Cells stably transfected with pcDNA3.1+ were seeded, and the medium was refreshed every other day with differentiation medium enriched with supplemented $\mathrm{CM}$ in a 2:1 v/v ratio. At days 1 and 21, cells were stored for Alizarin red staining and ALP activity assay. For hPDCs and hBMSCs differentiation with CM, the medium was refreshed every other day with differentiation medium enriched with supplemented CM derived from MC3T3-E1 cells stably transfected with pcDNA3.1+ control vector or pcDNA3.1Frzb. The supernatant was cleared by centrifugation at 3500 r.p.m. for $10 \mathrm{~min}$ and supplemented with $10 \mathrm{mM}$ $\beta$-glycerophosphate, $50 \mu \mathrm{g} / \mathrm{ml}$ L-ascorbic acid-2-phosphate and $0.1 \mathrm{nM}$ dexamethasone. The medium was refreshed every other day with differentiation medium enriched with supplemented CM in a 2:1 v/v ratio. At days 1 and 28, cells were stored for Alizarin red staining. Each condition was performed in triplicate.

\section{Western Blot Analysis}

MC3T3-E1 cells were harvested and resuspended in $0.05 \%$ Triton X (AppliChem, Zedelgem, Belgium) in PBS (Lonza, Verviers, Belgium) supplemented with $1 \mathrm{mM}$ 
GCATTCCCCTGTGCAAGT

AGCCCTGCAAGTCTGTGTGT

ATGGCGTCCTCTCTGCTTGA

AAGCAGGAGGGCAATAAGGT

CCACCGCGAGTGTGAGAT

TGCTGACCTGCTGGATTACA
GAGCAGTTCGAAGGTCTGCT

CCCCTCTGCAGTGTCCAGTA

AGTCCCGCAGAGGGCTAGAG

CAGGGTTAAGCTCACACTGCTC

ACATAGCCGGAACCTACGTG

TAGTCAGTTGCCCCCTGTAT phenylmethanesulfonyl (Sigma-Aldrich), 5\% protease inhibitor cocktail (Sigma-Aldrich), $2.3 \mathrm{mM} \quad \mathrm{Na}_{3} \mathrm{VO}_{4}$ (Sigma-Aldrich) and $5 \mathrm{mM} \mathrm{NaF}$ (Merck Millipore, Overijse, Belgium). Cell lysates were sonicated (two cycles of $7 \mathrm{~s}$ ) and centrifuged at 13000 r.p.m. for $10 \mathrm{~min}$, followed by protein quantification using the Pierce BCA Protein Assay kit (Thermo Scientific). A total of $7.5 \mu \mathrm{g}$ of protein of each sample was denatured and separated on a $4-12 \%$ polyacrylamide Bis-Tris gel (Invitrogen) by electrophoresis using NuPage MES SDS Running buffer (Invitrogen). Proteins were transferred onto a PVDF membrane (Merck Millipore). Non-specific binding sites were blocked using 5\% non-fat milk in Tris-buffered saline with $0.1 \%$ Tween (Applichem) $(\mathrm{TBS} / \mathrm{T})$ for $1 \mathrm{~h}$ at room temperature. Primary antibodies were incubated overnight at $4{ }^{\circ} \mathrm{C}$ with the following antibodies: $1 / 1000$ active (dephosphorylated) $\beta$-catenin (CTNNB1) mouse antibody (Millipore), 1/1000 total $\beta$-catenin mouse antibody (BD Biosciences, Aalst, Belgium), 1/1000 anti-pCamK rabbit antibody (Cell Signaling Technology, Leiden, The Netherlands), 1/1000 anti-CamK (pan) rabbit antibody (Cell Signaling Technology), 1/1000 anti-pCREB antibody (Cell Signaling Technology), 1/1000 anti-CREB antibody (Cell Signaling Technology), and $1 / 7500$ anti-actin rabbit antibody (housekeeping gene; Sigma-Aldrich) in 5\% bovine serum albumin in TBS/T (Cell Signaling Technology). Secondary horseradish peroxidaseconjugated antibodies were added, and blots were developed using SuperSignal West Femto Maximum Sensitivity Substrate (Pierce, Thermo Scientific). Densitometry of blots was performed using the Image J software (version 1.46r, National Institutes of Health) (Supplementary Tables 1 and 2).

\section{qPCR}

Total RNA was isolated using the NucleoSpin RNAII kit (Machery-Nagel, Düren, Germany) following the manufacturer's protocol. Complementary DNA was synthesized from 0.5 to $1 \mu \mathrm{g}$ of total RNA using the RevertAid H minus First Strand cDNA synthesis kit (Fermentas GmbH, Rockford, IL, USA). The SYBRgreen master mix system (Fermentas $\mathrm{GmbH}$ ) was used to verify differential expression of bFrzb, mFrzb, Osterix, Osteocalcin, and Axin2 (Table 1). SYBRgreen quantitative analysis was performed on the
Corbett Rotor-Gene 6000 (Corbett Research, Westburg, Leusden, The Netherlands) as follows: $10 \mathrm{~min}$ at $95^{\circ} \mathrm{C}, 40$ cycles of $15 \mathrm{~s}$ of denaturation at $95^{\circ} \mathrm{C}$, followed by $60 \mathrm{~s}$ of annealing-extension at $60^{\circ} \mathrm{C}$. Melting curve analysis was performed to ensure amplification of a specific product. Results are expressed using the comparative threshold method $^{14}$ and normalized to housekeeping gene Hprt1 (hypoxanthine guanine phosphoribosyl transferase1).

\section{ALP Activity}

Cells were resuspended in $0.05 \%$ Triton $\mathrm{X}$ in PBS and sonicated (two cycles of $7 \mathrm{~s}$ ). ALP activity was measured using the BluePhos Microwell Phosphatase Substrate System (KPL, Gaithersburg, MD, USA) following the manufacturer's instructions. Absorbance was measured at $495 \mathrm{~nm}$ with a Titertek Plus MS212 microplate reader (Tecan, Mechelen, Belgium). When absorbance reached the saturation level, samples were diluted 1 in 3 in $0.05 \%$ Triton $X$ in PBS.

\section{Alizarin Red Staining and Quantification}

At days 1, 7, 14, and 21, cells were washed with PBS and fixed with ice-cold 95\% methanol for $30 \mathrm{~min}$. Next, cells were washed with water and stained with $1 \%$ Alizarin Red (Sigma-Aldrich) at $\mathrm{pH} 4.1-4.3$ for 10-15 min. Three washing steps with water removed the unbound staining solution. Stains were dissolved in $10 \%$ acetic acid for $30 \mathrm{~min}$. Cells were scraped off the culture plates, vortexed and heated for $10 \mathrm{~min}$ at $85^{\circ} \mathrm{C}$. After $5 \mathrm{~min}$ on ice, the solution was centrifuged at 13000 r.p.m. for $15 \mathrm{~min}$ at $4^{\circ} \mathrm{C}$. $10 \%$ ammonium hydroxide was added to the aqueous phase in a 1:5 v/v ratio. Absorbance was measured at $405 \mathrm{~nm}$ with a Biotek Synergy HT microplate reader (Drogenbos, Belgium). When absorbance reached the saturation level, samples were diluted 1 in 3 in a mixture of $10 \%$ acetic acid and 10\% ammonium hydroxide (1:5 v/v ratio). The mineralization nodules in the hPDCS and hBSMCs CM experiments were quantified using the Image J software (NIH).

\section{Statistics}

Data were analyzed by two-way ANOVA taking into account repeated measurements as observations over time could not be considered independent. Time and Frzb expression were defined as factors. The overall effect of both factors and their 

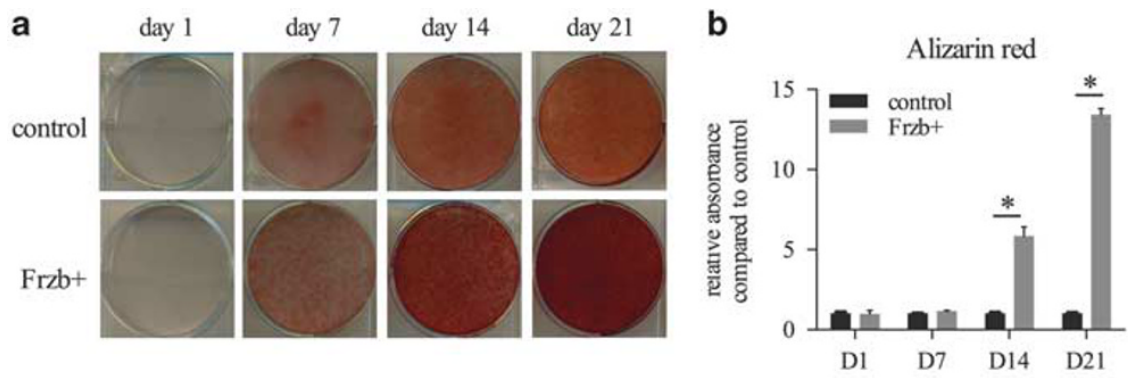

C
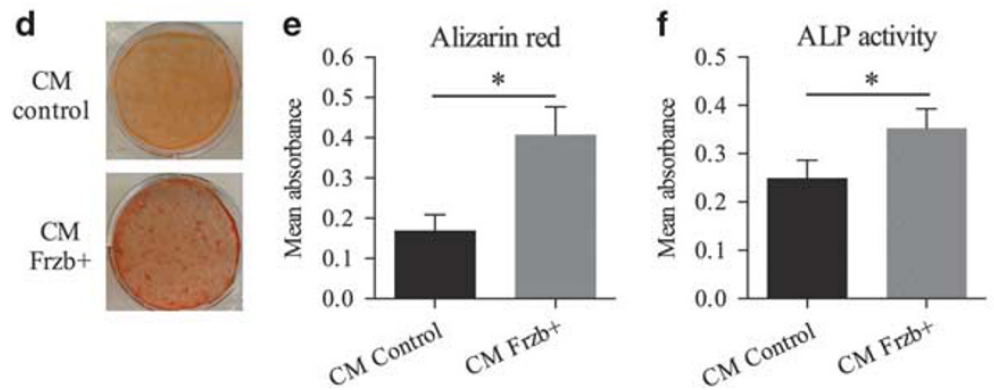

Figure 1 Overexpression of Frzb increases osteogenesis, and this effect is induced by secreted factors. (a) Alizarin red (AR) staining of MC3T3-E1 cells overexpressing Frzb (Frzb+) compared with controls. (b) Quantification of the AR staining of one in three diluted samples. Data are expressed as relative absorbance compared control with cells at the same timepoint (two-way ANOVA $P<0.0001$ for interaction between time and Frzb overexpression${ }^{*} P<0.05$ between control and overexpression). (c) Alkaline phosphatase (AP) activity of MC3T3-E1 cells overexpressing Frzb compared with controls (two-way ANOVA $P<0.0001$ for interaction between time and Frzb overexpression- ${ }^{*} P<0.05$ between control and overexpression). Data (a-c) show mean \pm s.d. of three replicates, but similar data were obtained in two additional experiments and for two other, independent cell lines overexpressing Frzb. MC3T3-E1 cells were stimulated with CM from Frzb overexpressing cells. (d) AR staining. (e) Quantification of the AR staining. Data are expressed as the mean absorbance. Unpaired $t$-test ${ }^{*} P=0.007$. (f) AP activity. Unpaired $t$-test ${ }^{*} P=0.03$. Data presented (d-f) are representative of two independent experiments with $\mathrm{CM}$ and show mean \pm s.d. of three independent replicates.

interaction was evaluated. Post hoc Sidak tests were applied for the different timepoints when interaction between time and target gene expression was found. Alpha was set at 0.05 .

\section{RESULTS}

\section{Overexpression of Frzb Increases Osteogenesis}

We studied the effects of FRZB on differentiation and mineralization in MC3T3-E1 cells, a mouse calvarial osteoblast precursor cell line. Stably transfected MC3T3-E1 cells were used, and high expression levels of Frzb were sustained throughout the 21-day experiments (average fold increase compared with control $3.5 \times 10^{3}$ ) (Figure 1a). Overexpression of Frzb resulted in significantly enhanced Alizarin red staining (two-way ANOVA $P<0.0001$ for interaction between time and Frzb overexpression $-P<0.05$ between control and overexpression at days 14 and 21, but not at days 1 and 7 , with difference between means respectively - 4.8 (95\% CI: $-5.5 ;-4,2)$ for day 14 and -12.4 (95\% CI: -13.0 ; 11.8) for day 21 (Sidak test)) (Figure 1a and b) and significantly increased ALP activity compared with control (two-way ANOVA $P<0.0001$ for interaction between time and Frzb overexpression $-P<0.05$ between control and overexpression at days $1,7,14$, and 21 , with difference between means respectively $-3.2(95 \%$ CI: $-6.0 ;-0.5)$ for day $1,-9.9(95 \%$ CI: $-12.6 ;-7.1)$ for day $7,-14.2$ (95\% CI: -16.9 ; -11.4 ) for day 14 , and -7.3 (95\% CI: -10.0 ; -4.5$)$ for day 21 (Sidak test)) (Figure 1c). Similar results were obtained with two additional and independent clones. Treatment of control MC3T3-E1 cells with CM from Frzb overexpressing cells increased both Alizarin Red staining (unpaired $t$-test $P=0.007$ ) (Figure $1 \mathrm{~d}$ and e) and ALP activity (unpaired $t$-test $P=0.03$ ) (Figure 1f) at day 21 .

\section{Overexpression of Frzb Modulates Wnt Signaling and Osteogenic Differentiation}

SFRPs were originally identified as inhibitors of Wnt signaling. Overexpression of Frzb decreased both active and total $\beta$-catenin. In contrast, Frzb overexpression increased the total amount and phosphorylation of CamKII and phosphorylated CREB, which is a downstream target of CamKII (Figure 2a). This suggests a negative effect on canonical signaling, but a positive effect on the non-canonical $\mathrm{Wnt} / \mathrm{Ca} 2+$ pathway.

Frzb overexpression decreased levels of Axin2, a known target gene in the canonical Wnt cascade (two-way ANOVA $P=0.0029$ for interaction between time and Frzb overexpression $-P<0.05$ between control and overexpression at days 14 and 21, with difference between means respectively 36.7 (95\% CI: 19.3; 54.0) for day 14 and 28.9 (95\% CI: 11.5; 46.2) for day 21 (Sidak test)) (Figure 2b). Gain of Frzb increased expression of osteoblast-specific transcription factors Osterix (two-way ANOVA $P<0.0001$ for interaction between 


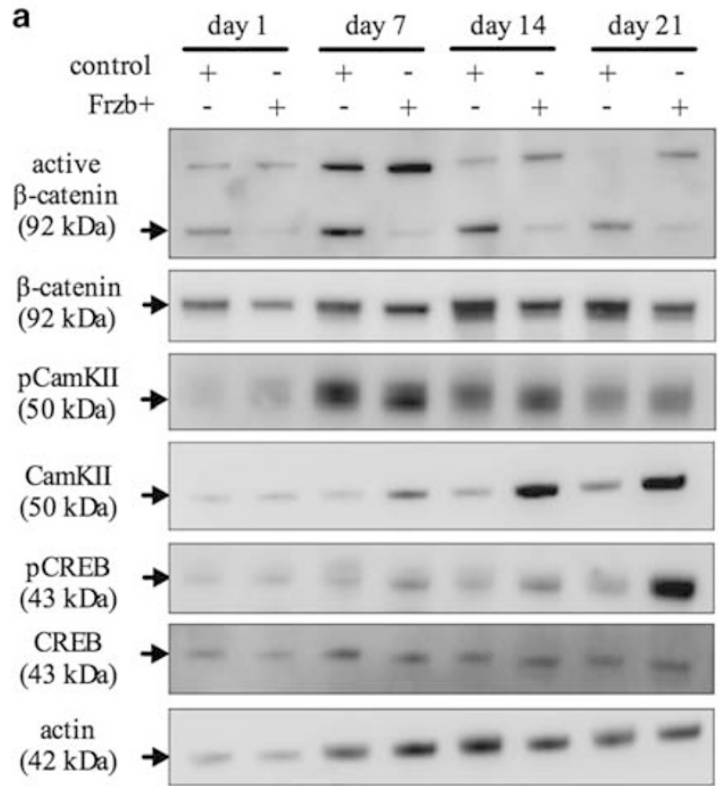

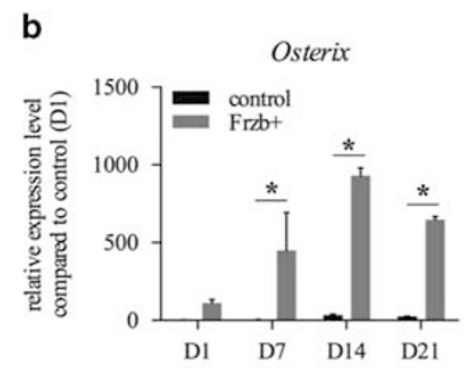
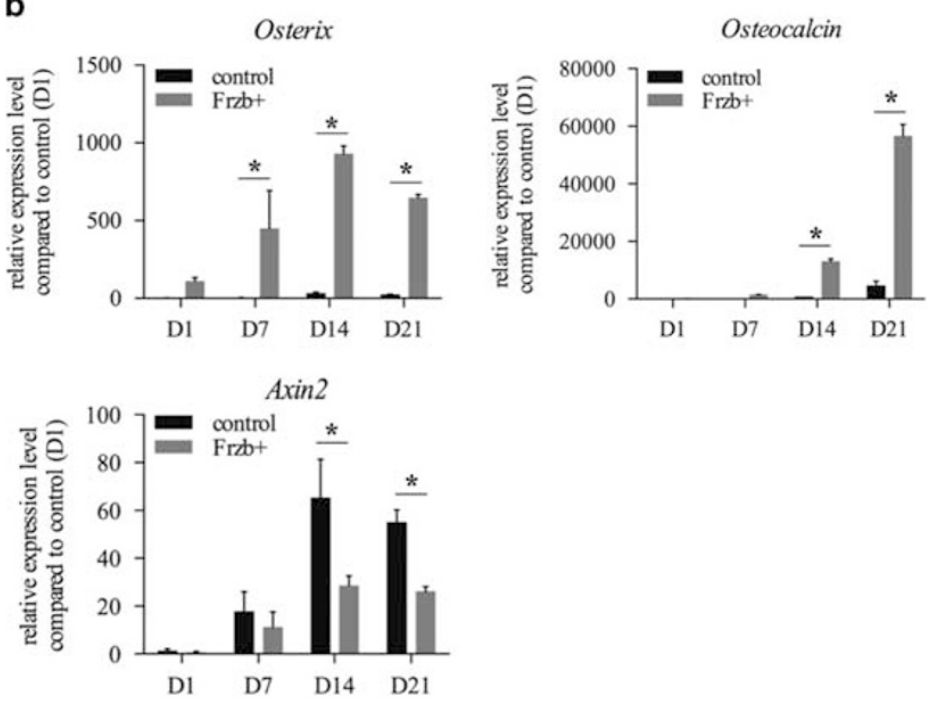

Figure 2 Overexpression of Frzb stimulates osteogenesis by decreasing canonical but stimulating non-canonical signaling. Western blot analysis shows a decrease in active (dephosphorylated) $\beta$-catenin $(92 \mathrm{kDa}$ ) and an increase in phosphorylated CamKII (50 kDa), total CamKII (50 kDa) and phosphorylated CREB (43 kDa) in Frzb (a) overexpressing cells. (b) mRNA expression levels of Osterix (two-way ANOVA $P<0.0001$ for interaction between time and Frzb overexpression $-{ }^{*} P<0.05$ between control and overexpression), Osteocalcin (two-way ANOVA $P<0.0001$ for interaction between time and Frzb overexpression - ${ }^{*} P<0.05$ between control and overexpression) and Axin2 (two-way ANOVA $P=0.0029$ for interaction between time and Frzb overexpression - ${ }^{*} P<0.05$ between control and overexpression) in MC3T3-E1 cells stably overexpressing Frzb (Frzb+). All mRNA levels were normalized to Hprt1 (reference gene) and shown as relative expression levels compared with control (D1). Data (a, b) show mean \pm s.d. of three replicates, but similar data were obtained in two additional experiments and for two other, independent cell lines overexpressing Frzb.

time and Frzb overexpression $-P<0.05$ between control and overexpression at days 7,14 , and 21 , with difference between means respectively $-446.5(95 \% \mathrm{CI}:-658.5 ;-234.6)$ for day 7 , -899.0 (95\% CI: $-1111 ;-687.1)$ for day 14 , and $-624.5(95 \%$ CI: -836.4 ; - 412.6) for day 21 (Sidak test)) and Osteocalcin (two-way ANOVA $P<0.0001$ for interaction between time and Frzb overexpression- $P<0.05$ between control and overexpression at days 14 and 21, with difference between means respectively - 12364 (95\% CI: - $16444 ;-8284)$ for day 14 and -52075 (95\% CI: -56 155; - 47 995) for day 21 (Sidak test)) (Figure 2b). Similar data were obtained in two additional experiments and for two other independent cell lines overexpressing Frzb.

\section{Loss of Frzb Negatively Affects Osteogenesis}

Silencing of endogenous Frzb throughout the 21-day experiment was obtained by a pGIPZ-shRNAmir plasmid directed against $F r z b(32,47,95$, and 97\% knockdown at days 1, 7, 14, and 21). Loss of Frzb decreased Alizarin red staining at day 14 and day 21 (two-way ANOVA $P=0.02$ for interaction between time and $F r z b$ silencing- $P<0.05$ between control and silencing at days 14 and 21, with difference between means respectively 0.6637 (95\% CI: $0.1 ; 1.2)$ for day 14 and 0.8520 (95\% CI: 0.3 ; 1.4) for day 21 (Sidak test)) (Figure 3a and b) and reduced ALP activity (two-way ANOVA $P<0.0001$ for interaction between time and $F r z b$ silencing $-P<0.05$ between control and silencing at days 14 and 21 , with difference between means respectively 3.6 (95\% CI: 3.3 ; 3.9) for day 14 and 8.358 (95\% CI: 8.0; 8.7) for day 21 (Sidak test)) (Figure 3c) compared with control shRNAmir. Decreased Frzb levels showed a reverse effect compared with overexpression on canonical and non-canonical cascades (Figure 3d). Loss of Frzb reduced expression of Osterix (two-way ANOVA $P<0.0001$ for interaction between time and $F r z b$ silencing $-P<0.05$ between control and silencing at days 14 and 21, with difference between means respectively 13.7 (95\% CI: 9.4; 17.9) for day 14 and 18.2 (95\% CI: 13.9; 22.4) for day 21 (Sidak test)) and Osteocalcin (two-way ANOVA $P<0.0001$ for interaction between time and Frzb silencing $-P<0.05$ between control and silencing at day 21 , with difference between means respectively 412.3 (95\% CI: 346.0; 478.6) for day 21 (Sidak test)), but did not affect Axin2 expression (Figure 3e). Similar data were obtained in an additional experiment and for an other independent cell line with loss of Frzb.

\section{Validation in Primary hPDCs and hBMSCs}

To translationally validate the positive effects of FRZB on primary human cells, we used hPDCs and hBMSCs, cell types with strong osteogenic potential. Treatment of hPDCs and hBMSCs with CM from MC3T3-E1 cells overexpressing Frzb increased Alizarin red staining at day 28 (unpaired $t$-test $P=0.03$ and $P=0.02$ ) (Figure 4). 
a

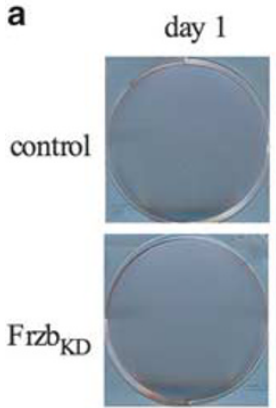

C

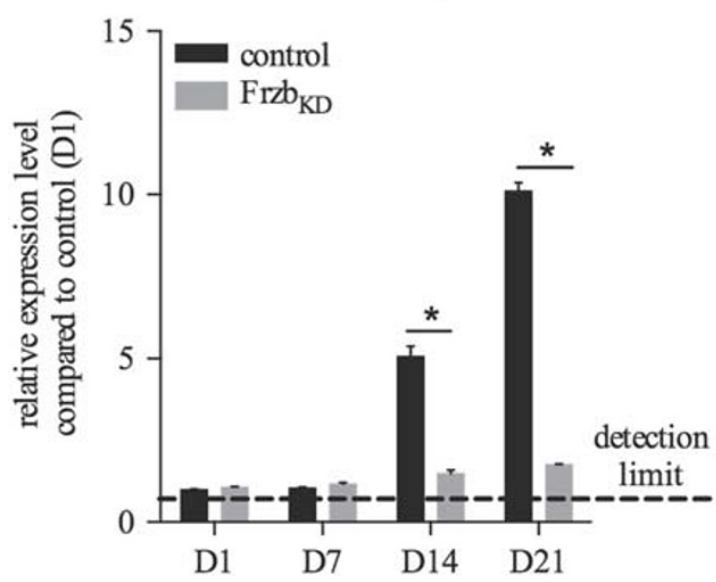

e

Osterix

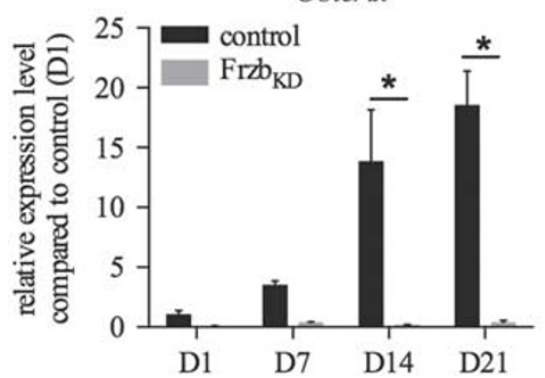

b

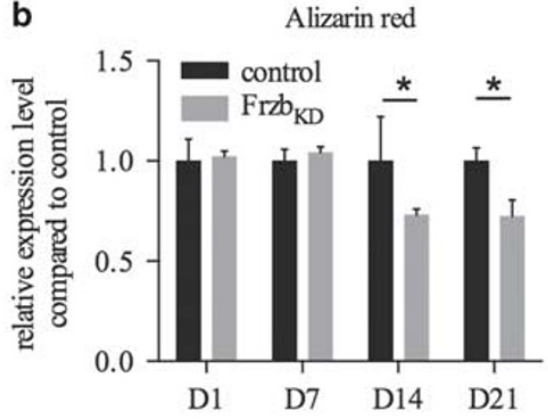

d
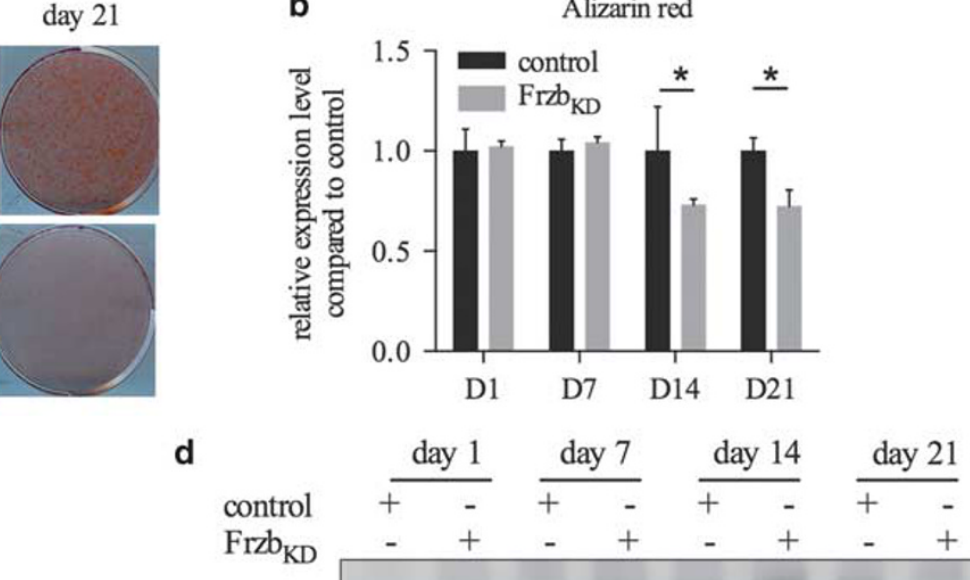

active

$\beta$-catenin

$(92 \mathrm{kDa}) \rightarrow$

$\beta$-catenin

(92 kDa)

pCamKII

(50 kDa)

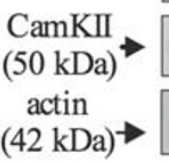

Osteocalcin

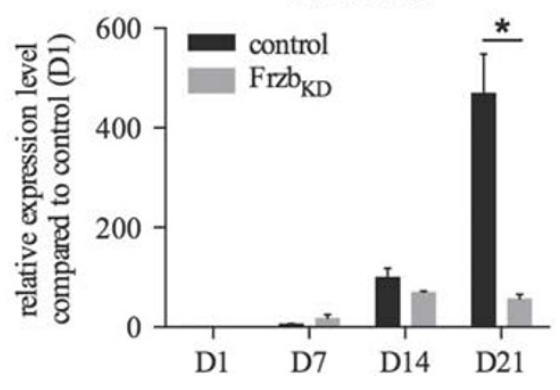

Figure 3 Knockdown of Frzb reduces osteogenesis. (a) Alizarin red (AR) staining of MC3T3-E1 cells in which Frzb is stably knocked down (Frzb compared with controls. (b) Quantification of the AR staining. Data are expressed as relative absorbance compared with control cells at the same timepoint. (two-way ANOVA $P=0.01$ for interaction between time and Frzb silencing ${ }^{*} P<0.05$ between control and silencing) (c) Alkaline phosphatase activity of Frzb $\mathrm{KD}_{\mathrm{D}}$ cells compared with control at day 1 (two-way ANOVA $P<0.0001$ for interaction between time and Frzb silencing- ${ }^{*} P<0.05$ between control and overexpression). (d) Western blot analysis shows a decrease in active (dephosphorylated) $\beta$-catenin (92 kDa) and increased phosphorylated

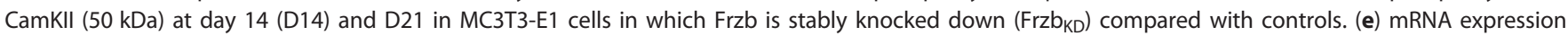
levels of Osterix (two-way ANOVA $P<0.0001$ for interaction between time and Frzb silencing- ${ }^{*} P<0.05$ between control and silencing), Osteocalcin (two-way ANOVA $P<0.0001$ for interaction between time and Frzb silencing- ${ }^{*} P<0.05$ between control and silencing), and Axin2 in Frzb $\mathrm{K}_{\mathrm{KD}}$ cells with loss of Frzb. mRNA levels were normalized to Hprt1 (reference gene) and shown as relative expression levels compared with control (D1). Data (a-e) show mean \pm s.d. of three replicates, but similar data were obtained in two additional experiments and for two other independent cell lines with loss of Frzb.

\section{Frzb $_{\text {NTN }}$ Overexpression Mimics Full-Length Frzb Overexpression in Osteogenesis}

We further studied the effect of the CRD and NTN motif in our assays. Sustained high expression of the Frzb $\mathrm{NTNN}_{\mathrm{N}}$ domain (average fold increase compared with control $3.1 \times 10^{3}$ ) increased Alizarin red staining (two-way ANOVA $P<0.0001$ for interaction between time and Frzb overexpression-
$P<0.05$ between control and overexpression at days 14 and 21 , with difference between means respectively -10.6 (95\% CI: -11.8 ; - 9.3) for day 14 and $-25.5(95 \%$ CI: $-26.8 ;-24.2)$ for day 21 (Sidak test)) (Figure $5 \mathrm{a}$ and c) and ALP activity (two-way ANOVA $P<0.0001$ for interaction between time and $\mathrm{Frzb}$ overexpression $-\mathrm{P}<0.05$ between control and overexpression at days $1,7,14$, and 21, with 
a

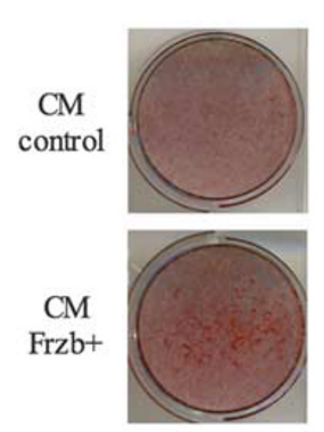

b

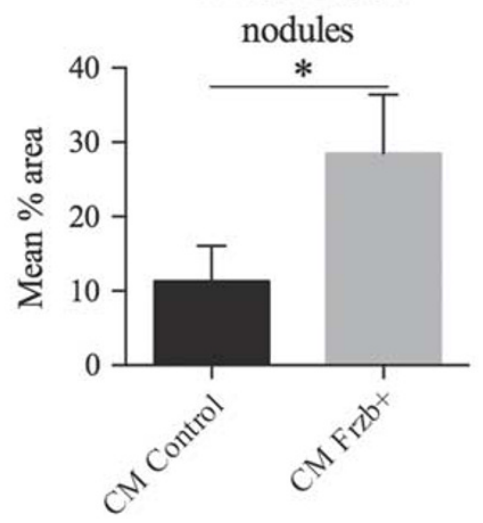

C

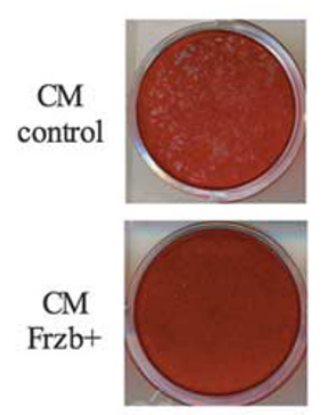

d

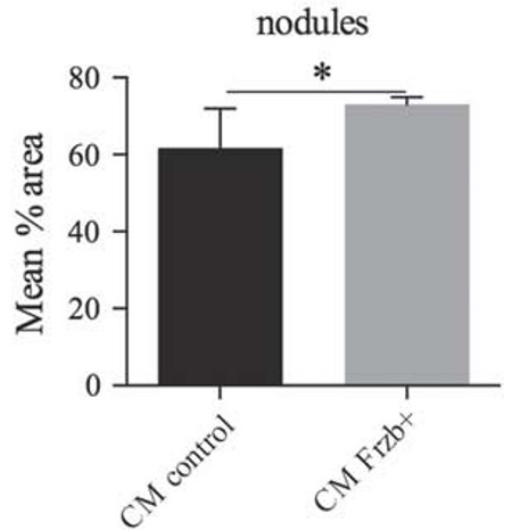

Figure 4 Positive effect of Frzb on osteogenesis in hPDCs and hBMSCs. (Left) Alizarin red (AR) staining of hPDCs (a) and hBMSCs (c) treated with CM of MC3T3-E1 cells stably overexpressing the control or Frzb+ construct at day 28 (D28). (Right) Quantification of the AR staining of hPDCs (b) and hBMSCs (d). Data are expressed as the percentage (\%) of the area that is covered by mineralized nodules. Data are shown as mean \pm s.d. of three (hPDCs) or six (hBMSCs) independent replicates. Unpaired $t$-test ${ }^{*} P=0.03$ and ${ }^{*} P=0.02$.

difference between means respectively -2.1 (95\% CI: -2.8 ; $-1.5)$ for day $1,-3.9(95 \% \mathrm{CI}:-4.5 ;-3.3)$ for day $7,-4.3$ (95\% CI: -4.9 ; -3.7 ) for day 14 , and -9.6 (95\% CI: -10.2 ; -9.0) for day 21 (Sidak test)) (Figure 5d). Additionally, gain of Frzb $\mathrm{NTN}_{\mathrm{NT}}$ increased Osterix expression at day 7 (two-way ANOVA $P=0.04$ for interaction between time and Frzb overexpression $-P<0.05$ between control and overexpression at day 7 with difference between means respectively - 132.0 (95\% CI: - 233.6; - 30.5) for day 7 (Sidak test)) (Figure 5e). Similar data were obtained in two additional experiments and for two other independent cell lines overexpressing Frzb $_{\text {NTN }}$. Treatment of hBMSCs with CM from MC3T3-E1 cells overexpressing $F r z b_{N T N}$ increased Alizarin red staining at day 28 (unpaired $t$-test $P=0.01$ ) (Figure 5b). On the contrary, overexpression of the Frzb ${ }_{\mathrm{CRD}}$ (average fold increase compared with control 75) domain slightly decreased Alizarin red staining (two-way ANOVA

$P=0.0007$ for interaction between time and Frzb overexpression $-P<0.05$ between control and overexpression at days 7 and 21, with difference between means respectively 0.2 (95\% CI: $0.09 ; 0.3$ ) for day 7 and 0.1 (95\% CI: 0.0002; 0.2) for day 21 (Sidak test)) (Figure $5 \mathrm{f}$ and $\mathrm{h}$ ) and ALP activity (two-way ANOVA $P<0.0001$ for interaction between time and $F r z b$ overexpression $-P<0.05$ between control and overexpression at days 14 and 21, with difference between means respectively 7.0 (95\% CI: $6.1 ; 7.9)$ for day 14 and 5.2 (95\% CI: 4.3; 6.1) for day 21 (Sidak test)) (Figure 5i). Moreover, the Frzb ${ }_{\mathrm{CRD}}$ domain did not consistently alter Osterix expression (Figure 5j). Similar data were obtained in two additional experiments and for two other independent cell lines overexpressing Frzb $\mathrm{C}_{\mathrm{CRD}}$. Frzb $\mathrm{b}_{\mathrm{NTN}}$ overexpression decreased active $\beta$-catenin and increased phosphorylated CamKII at day 21, while Frzb $b_{\mathrm{CRD}}$ overexpression decreased both active and total $\beta$-catenin throughout the whole experiment and decreased phosphorylated CamKII at day 21 (Figure 6a and b). Treatment of hBMSCs with CM from MC3T3-E1 cells overexpressing Frzb $b_{C R D}$ decreased Alizarin red staining at day 28 (unpaired $t$-test $P<0.0001$ ) (Figure $5 \mathrm{~g}$ ).

\section{Interaction of FrzbNTN and FrzbCRD with Full-Length Frzb and Wnts}

The observations reported above could indicate that Frzb ${ }_{\text {NTN }}$ and $\mathrm{Frzb}_{\mathrm{CRD}}$ have a dominant-negative effect on the fulllength protein. To further investigate potential competition of the overexpressed domains with the full-length protein, we treated MC3T3-E1 cells, control cells, and cells overexpressing Frzb $_{\mathrm{NTN}}$ or Frzb $\mathrm{C}_{\mathrm{CRD}}$ with recombinant mFrzb for 14 days. Adding recombinant FRZB to the differentiation culture slightly but significantly increased Alizarin Red staining in Frzb $_{\text {NTN }}$ overexpressing cells without a similar increase in ALP activity. In contrast, we did not detect a difference in Alizarin Red staining and ALP activity for control and Frzb $_{\text {CRD }}$ overexpressing cells after treatment with recombinant FRZB (Supplementary Figure 1A and C).

Next, we also studied the interactions between exogenously added Wnts and FRZB overexpressing cells. Wnt3a decreased Alizarin red staining in cells overexpressing Frzb and Frzb $_{\mathrm{NTN}^{+}}{ }^{+}$and decreased ALP activity in control cells and overexpressing Frzb. For Wnt5a, we could observe a decrease in Alizarin red staining for cells overexpressing Frzb, but we detected no differences in ALP activity (Supplementary Figure $2 \mathrm{~A}$ and $\mathrm{C})$. Moreover, treatment of MC3T3-E1 with recombinant mWnt3a resulted in an increase in active beta-catenin and phosphorylated CamKII in cells overexpressing Frzb and Frzb $\mathrm{NTN}_{\mathrm{N}}$, but in a decrease in canonical Wnt signaling in control cells. Addition of recombinant mWnt5a only positively influenced canonical Wnt signaling in cells overexpressing Frzb (Supplementary Figure 2D).

\section{DISCUSSION}

In our experiments, Frzb overexpression in vitro stimulated osteogenesis and was associated with reduced canonical and 
a
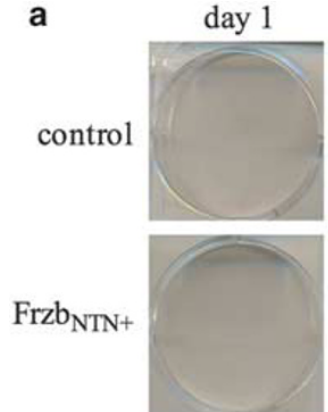

C

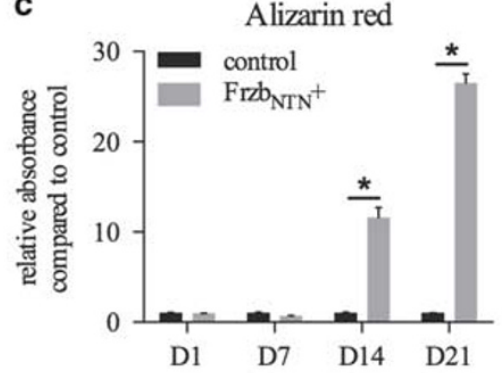

f
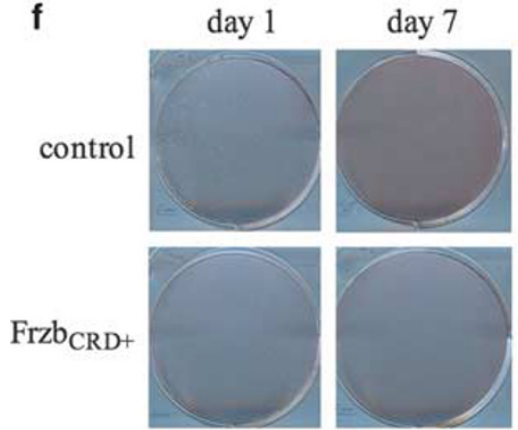

h

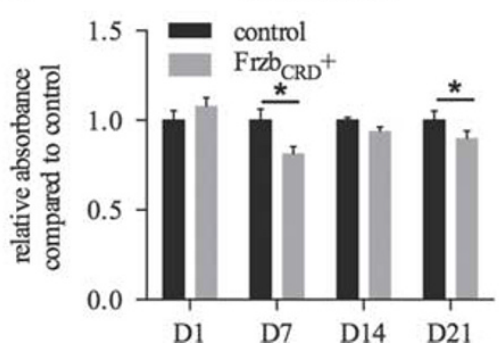

day 14

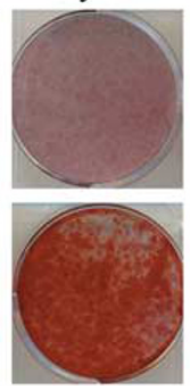

d day 21

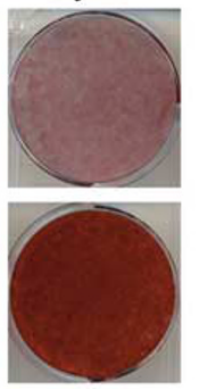

ALP activity b

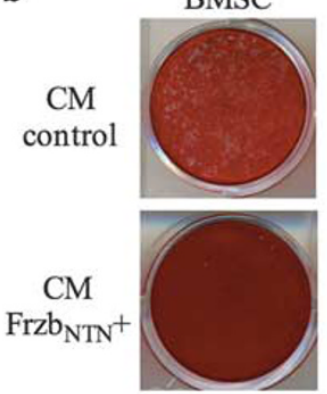

e

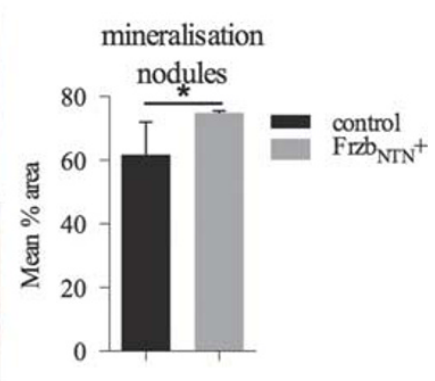

Osterix
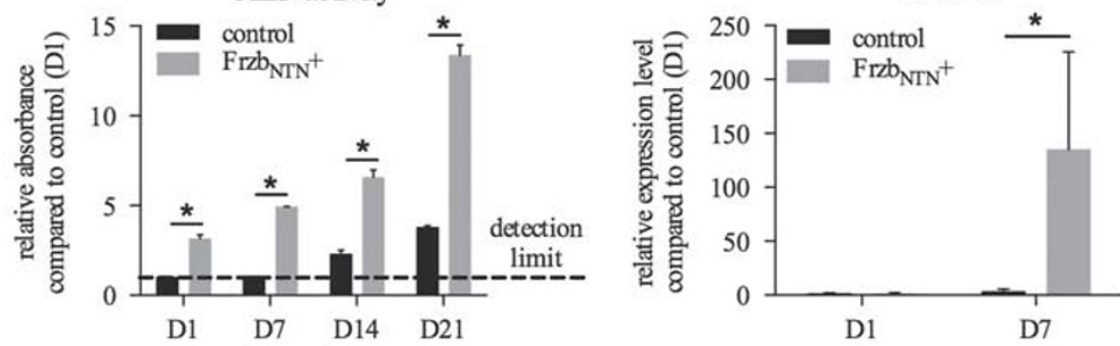

day 14

day 21
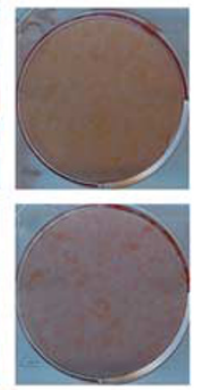

i

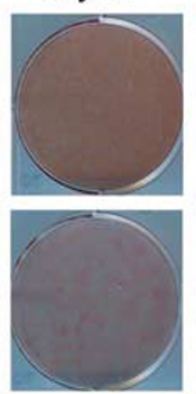

$\mathrm{g}$

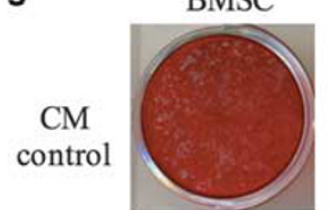

BMSC

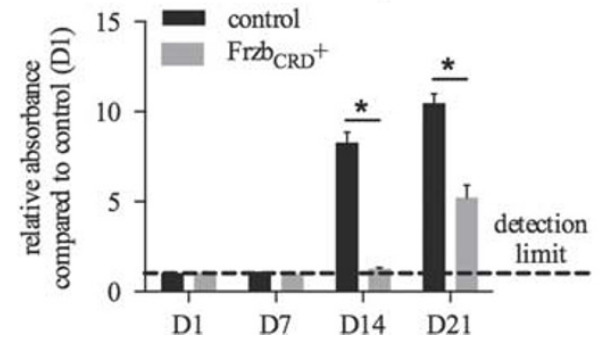

j

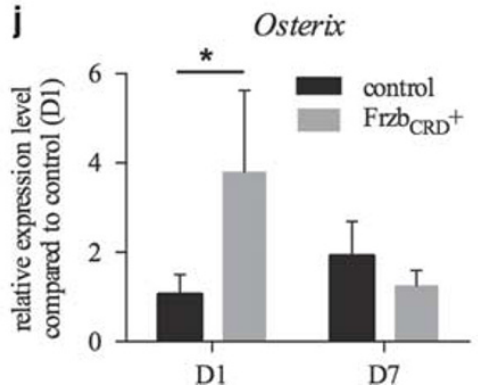

Figure 5 Frzb $_{C R D}$ and Frzb $b_{N T N}$ domains have different effects on osteogenesis. (a) Alizarin red (AR) staining of MC3T3-E1 cells with overexpressing Frzb $_{\text {NTN }}\left(\right.$ Frzb $_{\text {NTN }}+$ ) compared with controls. (b) AR staining of hBMSCs cells treated with CM of MC3T3-E1 cells stably overexpressing the control or Frzb $_{\text {NTN }}+$ construct at day 28 (D28) and quantification thereof. Data (b) are expressed as the percentage (\%) of the area that is covered by mineralized nodules. Data are shown as mean \pm s.d. of six independent replicates. Unpaired $t$-test ${ }^{*} P=0.01$. (c) Quantification of the AR staining. Data are expressed as relative absorbance compared with control cells at the same timepoint (two-way ANOVA $P<0.0001$ for interaction between time and Frzb overexpression - ${ }^{*} P<0.05$ between control and overexpression). (d) Alkaline phosphatase (AP) activity of Frzb $\mathrm{NTN}^{+}$cells compared with controls (two-way ANOVA $* P<0.0001$ for interaction between time and Frzb overexpression- ${ }^{*} P<0.05$ between control and overexpression). (e) mRNA expression levels of Osterix at day 1 (D1) and D7 (two-way ANOVA $P=0.0359$ for interaction between time and Frzb overexpression- ${ }^{*} P<0.05$ between control and overexpression). (f) AR staining of MC3T3-E1 cells overexpressing Frzb ${ }_{C R D}$ (Frzb CRD $^{+}$) compared with controls. (g) AR staining of hBMSCs cells treated with CM of MC3T3-E1 cells stably overexpressing the control or Frzb $\mathrm{CRD}+$ construct at D28 and quantification thereof. Data are expressed as the percentage (\%) of the area that is covered by mineralized nodules. Data are shown as mean \pm s.d. of six independent replicates. Unpaired $t$-test ${ }^{*} P<0.0001$. (h) Quantification of the AR staining (two-way ANOVA $P=0.0007$ for interaction between time and Frzb overexpression- ${ }^{*} P<0.05$ between control and overexpression). (i) AP activity of $\mathrm{Frzb}_{\mathrm{CRD}}+$ cells compared with controls at different timepoints (two-way ANOVA $P<0.0001$ for interaction between time and Frzb overexpression ${ }^{*} P<0.05$ between control and overexpression). (j) mRNA expression levels of Osterix at D1 and D7. mRNA levels were normalized to Hprt1 (reference gene) and shown as relative expression levels compared with control (D1). Data (a, c-f, h-j) show mean \pm s.d. of three replicates, but similar data were obtained in two additional experiments and for two other independent cell lines with loss of Frzb. 

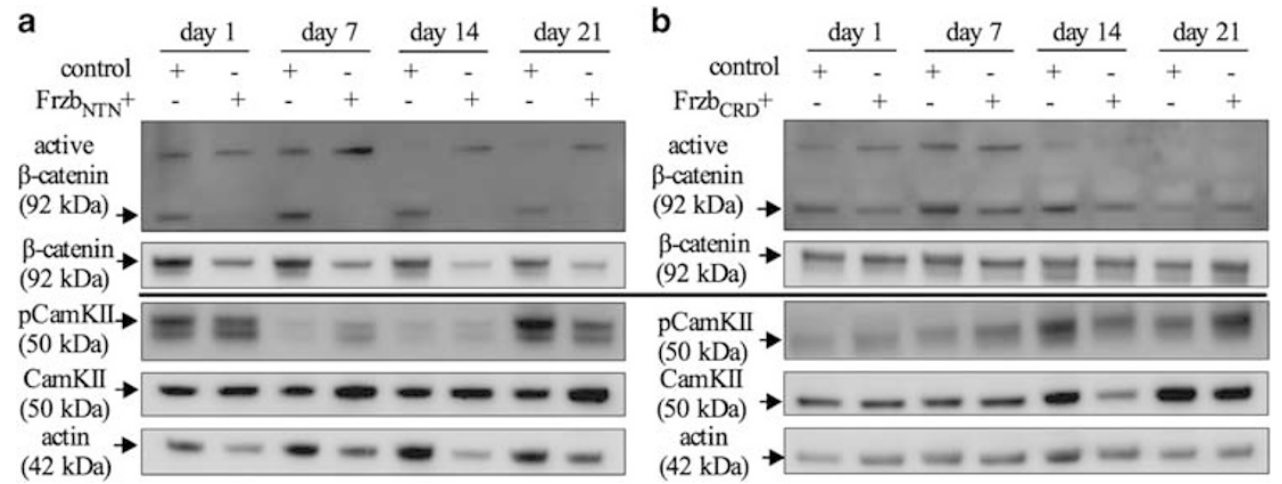

Figure 6 Both Frzb $\mathrm{CRD}_{\mathrm{CRD}}$ and Frzb $\mathrm{B}_{\mathrm{NTN}}$ overexpression decrease canonical Wnt signaling. (a) Western blot analysis shows a decrease in active (dephosphorylated) $\beta$-catenin $\left(92 \mathrm{kDa}\right.$ ) and a decrease in phosphorylated CamKII (50 kDa) in Frzb $b_{\text {NTN }}$ overexpressing cells at day 21. (b) Western blot analysis shows a decrease in active (dephosphorylated) $\beta$-catenin $(92 \mathrm{kDa}$ ) and an increase in phosphorylated CamKII $(50 \mathrm{kDa})$ in Frzb $\mathrm{CRD}$ overexpressing cells at day 21.

increased non-canonical Wnt signaling. On the other hand, loss of Frzb decreased osteogenesis. This observed positive effect on osteogenesis appears in line with earlier in vitro data, suggesting that recombinant Frzb increased osteoblast differentiation. ${ }^{15}$ However, these and our results appear contradictory to in vivo data showing that $\mathrm{Frzb}^{-1-}$ mice have increased cortical bone thickness, ${ }^{10}$ but not trabecular bone thickness and with the well-known observation that excess of Wnt signaling results in high bone mass phenotypes in mice and men. ${ }^{16-21}$ Other Wnt antagonists have also been shown to negatively affect bone formation. Genetic deletion of Sfrp1 in mice leads to an increased trabecular bone, while overexpression of SFRP1 inhibits bone formation. ${ }^{22-24}$ Furthermore, Sfrp1 acts as a negative regulator of human osteoblast and osteocyte survival and may also inhibit osteoclastogenesis by a Wnt-independent mechanism. ${ }^{22}$ Sfrp4 also negatively affects bone formation and SFRP4 was also proposed to be involved in phosphate metabolism, but others could not confirm this link. ${ }^{25,26}$ Additionally, loss of sclerostin (SOST) expression in bone was found to result in Van Buchem disease. ${ }^{27}$ In mice, targeted deletion of Sost results in increased bone formation and strength, while mice overexpressing Sost have low bone mass and decreased bone strength. ${ }^{28-30}$ Another Wnt antagonist, DKK1, regulates bone mass with increased expression linked to osteopenia and decreased expression associated with HBM. ${ }^{31}$ Dkk1 knockout mice are embryonic lethal, however, an allelic series of mice with decreased DKK1 expression levels $\left(D k k 1^{+/+}, D k k 1^{+/ d}\right.$, $D k k 1^{+/-}$, and $D k k 1^{d /-}$ ) show that for both the trabecular and the cortical bone, bone mass is inversely proportional to the level of DKK1. ${ }^{31}$ Interestingly, Dkk2, like Frzb, positively influences the formation of mineralized matrix in the late stages of osteogenic differentiation and this effect might not be entirely mediated by its Wnt signaling antagonistic activity. $^{32}$ We hypothesize that this surprising contrast between in vitro and in vivo observations can be explained by the high and therefore supraphysiological levels of the Wnt modulator. This overexpression may affect the protein's biological effects in different ways. Moreover, high local concentrations may be achieved in vivo in specific compartments of the skeleton but more importantly could be the goal of a treatment strategy. Frzb expression levels significantly increase during osteogenesis of MC3T3-E1 cells (Supplementary Figure 3), suggesting that Frzb has an important role in the later stages of osteogenesis and mineralization. This is in line with the expression profile of Dkk1, Dkk2, Sclerostin, and SFRP1, also Wnt inhibitors, during osteogenic differentiation..$^{22,32-34}$ Interestingly, while Sfrp1, Dkk1, and Sclerostin inhibit osteogenesis Dkk2 overexpression shows a similar effect as Frzb. ${ }^{33,34}$

Excess of canonical Wnt signaling results typically in high bone mass phenotypes in mice and men. ${ }^{16-21}$ However, in our gain of function studies, we observed a decrease in canonical Wnt signaling together with augmented osteogenesis and activation of the non-canonical Wnt/CamKII pathway. Although the effects of FRZB on osteogenesis can be mediated in part by its Wnt-inhibiting effect, we consider that the role of FRZB in osteoblast differentiation is not limited to its Wnt-antagonistic effect. First, our data suggest that canonical Wnt signaling is not the only mediator to drive osteogenesis in this in vitro system. The increase in osteogenesis could, at least partly, be caused by an increase in non-canonical Wnt signaling. $\mathrm{Ca}^{2+}$ signaling and $\mathrm{Ca}^{2+}$ transport have key roles during osteoblast differentiation and bone formation. Many of the cellular effects of $\mathrm{Ca}^{2+}$ are mediated by the $\mathrm{Ca}^{2+}$ binding protein, Calmodulin (CaM). Activation of CaM activates in turn both CamKII and CaN. ${ }^{35}$ Zayzafoon et al. ${ }^{36}$ showed that inhibition of $\alpha$ CamKII results in a decrease in osteoblast differentiation. Additionally, CamKII can stimulate osteoblastogenesis by modulating the activity of Osterix, a transcription factor essential for osteoblast differentiation and bone mineralization, and Dlx5, that mediates the transcriptional control by many osteoblastogenic signaling pathways. ${ }^{37,38}$ 
Second, although SFRPs were originally identified as soluble Wnt inhibitors that bind directly to Wnts and prevent their interaction with $\mathrm{Fz}$ receptors. However, different biochemical and crystallographic data suggested that SFRPs could both activate and inactivate Wnt signaling. SFRPs and $\mathrm{Fz}$ receptors have been shown to form homodimers and heterodimers via their CRDs and subsequently stimulate signal transduction. ${ }^{7}$ In our experimental setting Frzb overexpression inhibited canonical Wnt signaling, but stimulated non-canonical Wnt/Ca2+ signaling, by increasing both phosphorylated and total CamKII. Direct effects of modulator-receptor interaction therefore provide a possible explanation for our observations and require further research. Of note, Nalesso et al. ${ }^{39}$ demonstrated that distinct Wnt pathways are reciprocally inhibitory and in equilibrium and therefore selective inhibition of the canonical pathway enhances the activation of the $\mathrm{Ca}^{2+}$ mobilization. SFRPs have different binding affinities for distinct Wnt ligands. ${ }^{40}$ Canonical and non-canonical signaling pathway activation involves the shared use of the same Fz receptors. Therefore, excess of either type of Wnt might interfere with the other pathway as a result of ligand competition for binding to Fz. ${ }^{41}$ Third, SFRPs can interfere with protease activity, ${ }^{42}$ interact with proteins that are not directly involved in the Wnt signaling cascade, and affect BMP signaling. ${ }^{7,42-45}$

Frzb $_{N T N}$ overexpression induced similar effects on osteogenesis as full-length $F r z b$, whereas $F r z b_{C R D}$ overexpressing cells mimicked loss of Frzb experiments. The CRD of Sfrps is considered as the Wnt binding domain and is therefore most studied. Nevertheless, our data indicate that the NTN domain can also have strong effects on bone differentiation. Both the CRD and NTN domain are required for Wnt binding in Drosophila. ${ }^{9}$ We cannot exclude that the NTN domain has a role in Wnt binding. Earlier work found SNPs in the NTN domain associated with hip osteoarthritis. ${ }^{46}$ Additional data in this paper demonstrated that these variants in the NTN domain resulted in reduced Wnt-antagonistic activity for the full-length molecule. In addition, Wnt binding may not fully explain the observation that overexpression of Frzb $b_{\text {NTN }}$ or Frzb $_{\text {CRD }}$ resulted in a different outcome. The NTN domain shows sequence similarity to Netrin, TIMPs, and PCOLCEs. In TIMPs and PCOLCEs, the NTN domain is thought to interfere with protease activity. The Sfrp ${ }_{\mathrm{NTN}}$ domain may also have protease inhibitory activity. ${ }^{47}$ For example, SFRP2 can act as a procollagen C-proteinase enhancer of Tolloid-like metalloproteinases in myocardial infarction-associated fibrosis, ${ }^{48}$ SFRP1 can act as an inhibitor of the A Disintegrin and metalloproteinase domain-containing protein 10 metalloprotease, ${ }^{49}$ Sizzled, a non-mammalian Sfrp family member, stabilizes Chordin by binding and inhibiting the Tolloid-family metalloproteinase, Bmpla. ${ }^{43}$ Thus, even in a relatively straightforward in vitro setting FRZB and other SFRPs may have more complex biological effects than anticipated upon their discovery and initial functional assessment.
Although the use of stable cell lines is interesting from a practical point of view, this approach also has limitations. The exposure of the cells through their expansion to high levels of FRZB or modified forms of the molecule may affect their behavior in the described experiments. We also tested whether the mutated forms would have dominant-negative effects and how they interact with Wnt ligands. However,

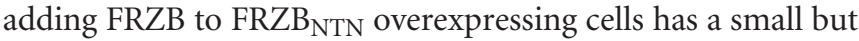
significant positive effect and not a competitive effect on osteogenesis. Wnt3a and Wnt5a do not seem to be able to interact with CRD without the NTN domain. However, FRZB and the FRZB $\mathrm{B}_{\mathrm{NTN}}$ appear to antagonize the inhibitory effect of Wnt3a on osteogenic differentiation.

In summary, our observation that overexpression of Frzb promotes bone differentiation and shifts balances between Wnt signaling cascades provides novel perspectives on the Wnt signaling pathway as a therapeutic target for bone and joint diseases. SFRPs or their specific domains may hold potential as therapeutics taking into account that our strong gain and loss of function approaches highlight that excess of SFRPs has effects that are not expected under physiological, endogenous expression conditions.

Supplementary Information accompanies the paper on the Laboratory Investigation website (http://www.laboratoryinvestigation.org)

\section{ACKNOWLEDGMENTS}

ST, FC and RL designed the project and wrote the manuscript. ST performed the experiments. ST, FC and RL analyzed the data. This work was supported by an OT ('Onderzoekstoelage') grant from KU Leuven and by FWO-Vlaanderen (Flanders Research Foundation) grants G.0470.10 and G.0A42.13.

\section{DISCLOSURE/CONFLICT OF INTEREST}

The authors declare no conflict of interest.

1. Westendorf J, Kahler RA, Schroeder TM. Wnt signaling in osteoblasts and bone diseases. Gene 2004;341:19-39.

2. Baron R, Kneissel M. WNT signaling in bone homeostasis and disease: from human mutations to treatments. Nat Med 2013;19:179-192.

3. Clevers $H$, Nusse R. Wnt/beta-catenin signaling and disease. Cell 2012;149:1192-1205.

4. MacDonald BT, Tamai K, He X. Wnt/beta-catenin signaling: components, mechanisms, and diseases. Dev Cell 2009;17:9-26.

5. Gao C, Chen YG. Dishevelled: the hub of Wnt signaling. Cell Signal 2010;22:717-727.

6. Cruciat CM, Niehrs C. Secreted and transmembrane wnt inhibitors and activators. Cold Spring Harb Perspect Biol 2013;5:a015081.

7. Bovolenta P, Esteve P, Ruiz JM et al. Beyond Wnt inhibition: new functions of secreted Frizzled-related proteins in development and disease. J Cell Sci 2008;121:737-746.

8. Gorny AK, Kaufmann LT, Swain RK et al. A secreted splice variant of the Xenopus frizzled-4 receptor is a biphasic modulator of Wnt signalling. Cell Commun Signal 2013;11:89.

9. Uren A, Reichsman F, Anest V et al. Secreted frizzled-related protein-1 binds directly to Wingless and is a biphasic modulator of Wnt signaling. J Biol Chem 2000;275:4374-4382.

10. Lories RJ, Peeters J, Bakker et al. Articular cartilage and biomechanical properties of the long bones in Frzb-knockout mice. Arthritis Rheum 2007;56:4095-4103.

11. Lin K, Wang $S$, Julius MA et al. The cysteine-rich frizzled domain of Frzb-1 is required and sufficient for modulation of Wnt signaling. Proc Natl Acad Sci USA 1997;94:11196-11200. 
12. De Bari C, Dell'Accio F, Luyten FP. Human periosteum-derived cells maintain phenotypic stability and chondrogenic potential throughout expansion regardless of donor age. Arthritis Rheum 2001;44:85-95.

13. Roobrouck VD, Clavel C, Jacobs SA et al. Differentiation potential of human postnatal mesenchymal stem cells, mesoangioblasts, and multipotent adult progenitor cells reflected in their transcriptome and partially influenced by the culture conditions. Stem Cells 2011;29: 871-882.

14. Giulietti A, Overbergh L, Valckx D et al. An overview of real-time quantitative PCR: applications to quantify cytokine gene expression. Methods 2001;25:386-401.

15. Chung YS, Baylink DJ, Srivastava AK et al. Effects of secreted frizzledrelated protein 3 on osteoblasts in vitro. J Bone Miner Res 2004;19: 1395-1402.

16. Boyden LM, Mao J, Belsky J et al. High bone density due to a mutation in LDL-receptor-related protein 5. N Engl J Med 2002;346:1513-1521.

17. Little RD, Carulli JP, Del Mastro RG et al. A mutation in the LDL receptor-related protein 5 gene results in the autosomal dominant high-bone-mass trait. Am J Hum Genet 2002;70:11-19.

18. Babij P, Zhao W, Small C et al. High bone mass in mice expressing a mutant LRP5 gene. J Bone Miner Res 2003;18:960-974.

19. Zhang Y, Wang Y, Li X et al. The LRP5 high-bone-mass G171V mutation disrupts LRP5 interaction with Mesd. Mol Cell Biol 2004;24:4677-4684.

20. Semenov MV, He X. LRP5 mutations linked to high bone mass diseases cause reduced LRP5 binding and inhibition by SOST. J Biol Chem 2006;281:38276-38284

21. Balemans W, Devogelaer JP, Cleiren E et al. Novel LRP5 missense mutation in a patient with a high bone mass phenotype results in decreased DKK1-mediated inhibition of Wnt signaling. J Bone Miner Res 2007;22:708-716.

22. Bodine PV, Billiard J, Moran RA et al. The Wnt antagonist secreted frizzled-related protein-1 controls osteoblast and osteocyte apoptosis. J Cell Biochem 2005;96:1212-1230.

23. Yao W, Cheng Z, Shahnazari $M$ et al. Overexpression of secreted frizzled-related protein 1 inhibits bone formation and attenuates parathyroid hormone bone anabolic effects. J Bone Miner Res 2010;25: 190-199.

24. Bodine PV, Zhao W, Kharode YP et al. The Wnt antagonist secreted frizzled-related protein-1 is a negative regulator of trabecular bone formation in adult mice. Mol Endocrinol 2004;18:1222-1237.

25. Cho HY, Choi HJ, Sun HJ et al. Transgenic mice overexpressing secreted frizzled-related proteins (sFRP)4 under the control of serum amyloid $\mathrm{P}$ promoter exhibit low bone mass but did not result in disturbed phosphate homeostasis. Bone 2010;47:263-271.

26. Nakanishi R, Akiyama H, Kimura $\mathrm{H}$ et al. Osteoblast-targeted expression of Sfrp4 in mice results in low bone mass. J Bone Miner Res 2008;23: 271-277.

27. Uitterlinden AG, Arp PP, Paeper BW et al. Polymorphisms in the sclerosteosis/van Buchem disease gene (SOST) region are associated with bone-mineral density in elderly whites. Am J Hum Genet 2004;75: 1032-1045.

28. Collette NM, Genetos DC, Economides AN et al. Targeted deletion of Sost distal enhancer increases bone formation and bone mass. Proc Natl Acad Sci USA 2012;109:14092-14097.

29. Moester MJ, Papapoulos SE, Lowik CW et al. Sclerostin: current knowledge and future perspectives. Calcif Tissue Int 2010;87:99-107.
30. Winkler DG, Sutherland MK, Geoghegan JC et al. Osteocyte control of bone formation via sclerostin, a novel BMP antagonist. EMBO J 2003;22:6267-6276.

31. MacDonald BT, Joiner DM, Oyserman SM et al. Bone mass is inversely proportional to Dkk1 levels in mice. Bone 2007;41:331-339.

32. Qiang YW, Barlogie B, Rudikoff S et al. Dkk1-induced inhibition of Wnt signaling in osteoblast differentiation is an underlying mechanism of bone loss in multiple myeloma. Bone 2008;42:669-680.

33. Li $\mathrm{X}$, Liu $\mathrm{P}$, Liu $\mathrm{W}$ et al. Dkk2 has a role in terminal osteoblast differentiation and mineralized matrix formation. Nat Genet 2005;37: 945-952.

34. Li X, Zhang $\mathrm{Y}$, Kang $\mathrm{H}$ et al. Sclerostin binds to LRP5/6 and antagonizes canonical Wnt signaling. J Biol Chem 2005;280:19883-19887.

35. Zayzafoon M. Calcium/calmodulin signaling controls osteoblast growth and differentiation. J Cell Biochem 2006;97:56-70.

36. Zayzafoon M, Fulzele K, McDonald JM. Calmodulin and calmodulindependent kinase Ilalpha regulate osteoblast differentiation by controlling c-fos expression. J Biol Chem 2005;280:7049-7059.

37. Seo $\mathrm{JH}$, Jin $\mathrm{YH}$, Jeong $\mathrm{HM}$ et al. Calmodulin-dependent kinase II regulates DIx5 during osteoblast differentiation. Biochem Biophys Res Commun 2009;384:100-104.

38. Choi $\mathrm{YH}, \mathrm{Choi} \mathrm{JH}, \mathrm{Oh} \mathrm{JW}$ et al. Calmodulin-dependent kinase II regulates osteoblast differentiation through regulation of Osterix. Biochem Biophys Res Commun 2013;432:248-255.

39. Nalesso G, Sherwood J, Bertrand J et al. WNT-3 A modulates articular chondrocyte phenotype by activating both canonical and noncanonical pathways. J Cell Biol 2011;193:551-564.

40. Wawrzak D, Metioui M, Willems E et al. Wnt3a binds to several sFRPs in the nanomolar range. Biochem Biophys Res Commun 2007;357:1119-1123.

41. Grumolato L, Liu G, Mong $P$ et al. Canonical and noncanonical Wnts use a common mechanism to activate completely unrelated coreceptors. Genes Dev 2010;24:2517-2530.

42. Lee $\mathrm{HX}$, Ambrosio AL, Reversade $\mathrm{B}$ et al. Embryonic dorsal-ventral signaling: secreted frizzled-related proteins as inhibitors of tolloid proteinases. Cell 2006;124:147-159.

43. Muraoka O, Shimizu T, Yabe T et al. Sizzled controls dorso-ventral polarity by repressing cleavage of the Chordin protein. Nat Cell Biol 2006;8:329-338.

44. Yabe T, Shimizu T, Muraoka $\mathrm{O}$ et al. Ogon/Secreted Frizzled functions as a negative feedback regulator of $\mathrm{Bmp}$ signaling. Development 2003;130:2705-2716.

45. Misra K, Matise MP. A critical role for sFRP proteins in maintaining caudal neural tube closure in mice via inhibition of BMP signaling. Dev Biol 2010;337:74-83.

46. Loughlin J, Dowling B, Chapman K et al. Functional variants within the secreted frizzled-related protein 3 gene are associated with hip osteoarthritis in females. Proc Natl Acad Sci USA 2004;101:9757-9762.

47. Chong JM, Uren A, Rubin JS et al. Disulfide bond assignments of secreted Frizzled-related protein-1 provide insights about Frizzled homology and netrin modules. J Biol Chem 2002;277:5134-5144.

48. Kobayashi K, Luo M, Zhang $Y$ et al. Secreted Frizzled-related protein 2 is a procollagen $C$ proteinase enhancer with a role in fibrosis associated with myocardial infarction. Nat Cell Biol 2009;11:46-55.

49. Esteve $\mathrm{P}$, Sandonis $\mathrm{A}$, Cardozo $\mathrm{M}$ et al. SFRPs act as negative modulators of ADAM10 to regulate retinal neurogenesis. Nat Neurosci $2011 ; 14: 562-569$. 